\title{
The Emergence and Dynamics of Electronic Health Records - A Longitudinal Case Analysis of Multi-Sided Platforms from an Interoperability Perspective
}

\author{
Lars Stegemann \\ Freie Universität Berlin, Germany \\ lars.stegemann@fu-berlin.de
}

\author{
Martin Gersch \\ Freie Universität Berlin, Germany \\ martin.gersch@fu-berlin.de
}

\begin{abstract}
Emerging health record platforms are interesting examples of the ongoing process of digitalization and the great opportunities they provide for innovation and additional services. Incumbent players are under increasing pressure from new entrants to offer their customers a user experience they have become familiar with through platforms such as Apple and Google. The emergence of the digital German health record is shown as a case-study, harnessing a longitudinal database and adopting a processsensitive perspective. Important events are structured into individual episodes and phases and discussed indepth. The study shows how platform owners of health records respond to changes in the highly regulated healthcare system and its digitalization in Germany. Contrasting with extant knowledge about interoperability as a relevant precondition for platforms, our study shows the important role played by interoperability as a design parameter for emerging platforms, which results in seven interoperability challenges for respective stakeholders.
\end{abstract}

\section{Introduction}

Compared to other industries, the progress of digitalization is much slower in the healthcare sector [16]. Reasons include the necessary regulation within divergent national contextual frameworks, the presumption of market failure, the complex systems of care and treatment processes, the variety of stakeholders and heterogeneous systems and the lack of interoperability (e.g., [19]). One of the key applications, and the basis for various add-on services in healthcare, is the Electronic Health Record (EHR), which drives and underpins the digitalization of healthcare. Due to the slow progress of digitalization, the healthcare market still offers great potential for the development of innovative services [16]. The chairman of the board of the Techniker Krankenkasse (TK), one of the leading statutory health insurance companies in Germany, communicated this very clearly in his vision for the TK at a key speech in October 2019:

"TKs vision for 2030 is shaped by the conviction that people live in a few, relatively stable digital ecosystems with high levels of connectivity. Besides Apple and Amazon, a healthcare ecosystem is to be designed by TK with a quality and user experience on a par with Apple \& Co. In this context, the regulatory authorities in Germany will hopefully prevent statutory health insurance companies from losing direct contact with the customer during the transition phase as well. The TK ecosystem will include databased services beyond the mandatory requirements (e.g., electronic patient files) and will persuade and inspire the loyalty of TK-insured persons in the long term. We will clearly use digitization to differentiate ourselves from the competition in order to achieve greater control of the provision of care ${ }^{\prime \prime}$ (Thomas Ballast 2019; Board member TK, Berlin \#eHealthCon October 23, 2019).

In addition to this particular vision for the healthcare sector by this insurance company, the quote also elucidates the threat of new market entrants that it will have to deal with and how it will address these threats in terms of quality, user experience and service offerings. Health record platforms play a central role in this context. In order to develop the full potential of EHR, semantic interoperability, in addition to technical specifications, must be achieved despite its being one of the most challenging tasks in health informatics (e.g., [21]). Especially in the case of providers of national EHRs, such as for Denmark or Norway, a clear consensus about standards is necessary to ensure the proper exchange of information between different healthcare service providers and sectors in order to realize the benefits of platform-based coordination [14, 19, 1]. Germany's

\footnotetext{
${ }^{1}$ Author's translation from German into English
} 
self-administered health system (consisting of associations, institutes and organizations of stakeholders in the health sector e.g., [23]) has not been able to reach a consensus of interoperability issues for decades. In 2019 and 2020, comprehensive changes have taken effect in order to regulate the digitalization of the German healthcare system records and promote innovative health services. This refers in particular to EHRs: by January 1, 2021, all statutory health insurance companies are required by law to provide their policyholders with an EHR. As of June 2020, six potential providers - two insurance companies, Allgemeine Ortskrankenkasse (AOK) and $\mathrm{TK}$, and four service providers (VIVY, IBM, Compugroup Medical and RISE)-have begun developing an EHR in response to governmental specifications. In the German context, health records can be differentiated into one of two types, as shown in Table 1. On the one hand, the EHR represents the governmental required minimal standard of a repository to store and exchange health status information, which is securely stored and shared with other institutions, based on the specifications of the regulatory authorities. The EHR facilitates the longitudinal sharing of medically relevant data, e.g., diagnosis, treatment activities, laboratory data and radiology reports, which can be exchanged among different health service providers across organizations $[4,19]$ and is primarily administered by physicians. On the other hand are provider-specific Personal Health Records (PHR) which include personal health information and which are primarily administered by the user [4, 19]. PHRs are an additional optional service of the EHR and provide the basis for data generation as well as complementary offers of mostly data-driven services within emerging providerspecific health ecosystems. In this context, and as shown by Ballast's quote above, it is in the interest of providers such as TK and AOK to establish multisided health platforms, especially around the PHR, in order to offer a competitive additional benefit to interested insurants, by e.g. exclusive complementors and their digital services. Both records will be managed by the provider/platform owner, but in contrast to the PHR, the EHR will have to follow government specifications and regulations. Regarding PHRs, each of the platform owners address different interests and follow different design approaches over the course of implementation as well as different government structures and rules, e.g., relating to openness [31] of their boundary resources (e.g., [13]). Despite the fact that the EHR will only become mandatory in 2021, competition between platforms has already begun. In terms of interoperability, these government requirements impose various challenges for platform providers and platform owners. This leads to the following two research questions:

$R Q$ 1: How do PHR and their respective ecosystem develop differently over time in a highly regulated market?

$R Q$ 2: Which specific interoperability challenges can be observed prior to the official implementation of EHRs in Germany?

In order to answer these questions, this study follows a research design in digital transformation and platformization (e.g., [11, 26]) in form of a longitudinal, processsensitive, comparative, embedded case study [9, 33]. The case will be analyzed on the basis of primary data (e.g., fieldnotes and interviews) and secondary data (e.g., press releases, professional articles, legal reforms) which will be compiled together in a digital case study database following Yin's approach [33]. In research projects with long-term data, it is particularly important to observe the research phenomenon as a changing process over the development of the research project; however, there are very few precedents of this type of research (e.g., [11]). In the context of multisided platforms (MSP), Fürstenau et al. [11] developed a design and management framework, which will be used to analyze and compare the differences between three selected PHR platforms in Germany during the emergence process. The framework was developed by Fürstenau et al. [11] as an extension of the integrative framework for platforms by Gawer \& Cusumano [12]. It specifies the four strategic design dimensions (issues) manifested over the course of platform development and contextualizes the design of interoperability as an embedded and interdependent design parameter.

\section{Conceptual Background}

\subsection{Multi-Sided Platforms in Germany}

Besides the described differences between PHRs and EHRs, another differentiation exists between government-regulated or open solutions [6], on a regional, national or institutional level. Additionally, the storage location of health records can be either

Table 1: Types of health records in Germany

\begin{tabular}{|l|l|l|l|}
\hline Type & Regulation & \multicolumn{1}{l|}{$\begin{array}{l}\text { Provider/ } \\
\text { Platform } \\
\text { owner }\end{array}$} & Storage Location \\
\hline EHR & $\begin{array}{l}\text { Governmental } \\
\text { regulation }\end{array}$ & $\begin{array}{l}\text { Insurance } \\
\text { companies }\end{array}$ & $\begin{array}{l}\text { Decentralized by } \\
\text { the service } \\
\text { provider }\end{array}$ \\
\hline PHR & $\begin{array}{l}\text { By the } \\
\text { platform } \\
\text { owner }\end{array}$ & $\begin{array}{l}\text { Insurance } \\
\text { companies } \\
\text { or free } \\
\text { provider }\end{array}$ & $\begin{array}{l}\text { Centralized or } \\
\text { decentralized } \\
\text { depending on the } \\
\text { platform owner }\end{array}$ \\
\hline
\end{tabular}


centralized or decentralized (see Table 1). In Germany, the EHR will be a national, decentralized, government-regulated solution, which means that the data will be stored in the "primary information system" of the service provider who generates the EHR data. Other authorized service providers throughout the nation can retrieve requested health data. The data will be shared via the German health information exchange infrastructure, which is a nationwide secure Virtual Private Network (VPN), called Telematic Infrastructure (TI). The technical background processes as well as the user interface are supplied by the EHR provider (see Table 1) via the TI. Gematik as the lead organization is responsible for the technical specifications, standards, testing and operations of the TI. In contrast to the EHR, the PHR is specified by the providers or platform owners. Given that many different independent stakeholder groups - medical service providers, complementors of digital services, and patients - are involved, PHR Platforms can be understood as an MSP [5, 19, 32]. The success of MSPs is significantly influenced by network effects and a highly dynamic ecosystem [8, 26]. Kapoor [18] understands an ecosystem as "a set of actors that contribute to the focal offer's user value proposition" [18 p. 2]. Its actors would include, e.g., participating physicians, complementors of digital health services, insurants, and the insurance company. Concerning platforms and their respective ecosystems, Hein et al. [15] identified three central building blocks of a digital platform ecosystem, consisting of platform owner, value-creation mechanism and complementor. The value-creation mechanism facilitates the joint creation of value by the platform owner and complementors and provides a basis for promoting innovation within the platform ecosystem [15]. The platform owner basically determines the design, resources and management of the platform ecosystem [15] according to Fürstenau et al.'s [11] design and management framework. Choosing a mode of governance [26] is the responsibility of the platform owner, who also promotes innovation for the platform ecosystem, especially by third-parties who are respectively known as complementors [5].

\subsection{Interoperability}

Our understanding of interoperability is related to the approach of the IEEE [17]. For MSPs, such as PHR platforms, interoperability is an essential precondition for exchanging data $[10,4,3]$. One requirement of Fürstenau et al.'s [11] framework, and also a key competitive parameter, is that the platform must be able to achieve direct network effects through standards and interfaces [8] as well as competitive advantages through the design of the integration and binding of complementors and customers. This requires a certain degree of openness/closeness (e.g., [31]) within the platform's ecosystem, which can be achieved through the definition of governance rules for the boundary resources (e.g., [13]) and the selection of interface standards [27, 11]. In general, interoperability can be achieved through the use of standards, especially open standards. These standards can be assigned to different levels of interoperability according to their respective types. A distinction is made between technical, syntactical, semantical and pragmatic levels (e.g., [3, 25]). In order to implement a PHR/EHR system, the technical level will be represented by the IT architecture as well as its compliance to the TI. Syntactical and semantical interoperability refers to the format, especially regarding the ease of understanding the transmitted message. In order to benefit from the potential of health records (e.g., big data and machine learning algorithms), semantically interoperable data are required [21], i.e., the exchanged information must be able to be uniformly interpreted and understood (e.g., $[3,25])$.

\section{Method}

\subsection{Research design}

In order to answer the research questions, an essential understanding about the process and events is required, especially regarding the regulatory changes that will occur over the course of the emergence of the EHR and PHR platforms in Germany. These are part of an ongoing parallel and sequential chain of events. We understand events to be actions, reactions or decisions made by the platform owners, conceivable complementors (stakeholders) or government. Episodes are sequences of changes, e.g., by government, and resulting actions, reactions or decisions made by platform owners or complementors, as well as actions that occur during the process of the platform's emergence. The dissemination of health platforms is dynamic, context-sensitive, and timespecific and requires a longitudinal analysis study [32]. For a more nuanced understanding, the events can be considered on different levels as well as in the context of different issues in terms of platformization. Therefore, we have chosen a longitudinal, processsensitive and comparative perspective with an indepth view of platformization according to the platform management framework by Fürstenau et al. [11]. The overall study is designed as embedded case study following Eisenhardt [9] and Yin [33] and focusses on contemporary events. 


\subsection{Case selection and data collection}

Germany has one of the oldest healthcare systems in the world. It follows a solidarity principle where every citizen is insured by one of the statutory health insurance providers (numbering 105, as of January 1, 2020), with the exception of certain professional groups (e.g., soldiers, public servants, freelancers, or high earners). Measured in terms of GDP, Germany has the third highest health expenditure [24]. A deeper overview of the German healthcare system is provided by Obermann et al. [23] and Busse et al. [7]. Our study will focus on three emerging PHR platforms with the highest number of potential users and some interesting strategic differences. Two of the platform owners belong to the largest statutory health insurance companies in Germany, the AOK and the TK. In contrast to these incumbent companies, the third platform in our study is the entrant VIVY, a private company that offers a white label solution for statutory health insurance as well as for private insurance companies (see also [2]). Due to the high potential number of users and the significant differences in their PHR solutions, especially in terms of architecture, these three providers are compared in a comparative case study approach [33]. While designing our case study, we reconstructed the process of episodes as well as the relevant events involving the respective actors, including regulatory changes over time, and thus the emergence of the PHR platforms in Germany. We used different sources of data to avoid potential biases within the primary data, and triangulate the results obtained from different sources of secondary data. Table 2 presents an overview of the data used in the case study database [33].

\subsection{Data preparation and analysis}

The collected data is coded and structured by level of analysis, time stamp and influence directions according to the management framework developed by Fürstenau et al. [11]. Additionally, the temporal bracketing approach by Langley [20] is used to identify relevant events and episodes in the data. This structure enables a differentiation of the data and the selection of decisive events during the emergence of the EHR as well as the PHR platforms. In a further conceptualization, we distinguish between the upcoming events of different, partly interdependent levels of analysis in terms of the impact on the macro, meso or micro level (e.g., [22]). Events with a wide impact on the overall healthcare system can be assigned to the macro level, e.g., regulatory changes made by government. In contrast, the micro level will include events with a limited scope and impact, such as in a single organization or a small focus group. The meso level represents events in between the two, i.e., events orchestrated by the platform owner for their ecosystem or for their insurants. Events on this level have a wider impact on all their insurants and/or the respective ecosystem of the complementors of the emerging PHR platforms.

In terms of the emergence of the case, we identified three overall phases for an initial structuring of the information on the macro level in the data. During the first Phase, I Experimental PHR phase, the insurance companies in particular were able to gain initial experience with a PHR. In the second phase, II Transition phase from PHR to EHR, it became obvious that an EHR will become mandatory according to specifications. In the third phase, III EHR becomes mandatory, the EHR will be introduced in Germany (on January 1, 2021). After this date, every statutory health insurance company must offer an EHR to its insurants. To structure and reconstruct the events and episodes of the platform's emergence, we follow the four "issues" of Fürstenau et al.'s [11] platform design and management framework. The issues follow a platform management point of view on the micro and meso level relating to the platform owner. The first issue, 1) Developing strategy and governance model, refers to decisions about the vision and governance structures of the platform. Decisions about the architecture and interoperability of the platform can be coded as being part of the second issue, 2) Designing technical architecture and selecting standards. The third issue, 3) Facilitating participation and community building, refers to actions and decisions related to developing the community around the platform ecosystem. Decisions to form alliances with

Table 2: Case study database

\begin{tabular}{|l|l|c|c|}
\hline Document type & Document description & Documents & Pages \\
\hline $\begin{array}{l}\text { Participatory } \\
\text { observations }\end{array}$ & $\begin{array}{l}\text { Field notes from various events, including lectures and discussions by } \\
\text { responsible stakeholder (from November 2018-May 2020) }\end{array}$ & 31 & 112 \\
\hline Interviews & $\begin{array}{l}\text { Semi-structured and formally recorded and transcribed interviews with health } \\
\text { startups (collected in 2019 as a pre-study) }\end{array}$ & 7 & 72 \\
\hline Press releases & Press releases, position papers, presentation slides, blog articles & 59 & 246 \\
\hline Professional articles & Articles from professional digital health journals & 28 & 212 \\
\hline Legal reforms & $\begin{array}{l}\text { Legal reforms to digitize the German healthcare (2004-2020) } \\
\text { Statements about the reforms that provide background information }\end{array}$ & 6 & 598 \\
\hline Specifications & Specification documents by regulatory authorities & 6 & 422 \\
\hline
\end{tabular}


platforms or authorities can be assigned to the last issue, 4) Engaging with the platform's ecosystem and wider environment. By structuring the data as described, patterns and causal relationships can be systematically established to reconstruct the case and understand the impact of partially interlinked events i.e. actions, reactions and decisions.

\subsection{Case analysis of the emergence of health record platforms in Germany}

3.4.1. Introduction to the case analysis. This section shows the dynamics within the emergence of the three focused PHRs/EHRs in Germany over a period of sixteen years. Starting from the regulatory changes on the macro level, the three PHR platforms on the micro and meso levels will be briefly explained. For this purpose, the events are categorized into the three distinct phases as well as the four issues identified by Fürstenau et al. [11]. Figure 1 gives an overview, with more details provided in the following subsections.

3.4.2. The case from the macro level point of view. Initially, the emergence of health records can be described from a macro perspective, i.e., essential events, particularly governmental regulations and legislation as well as the establishment of infrastructure and the definition and selection of standards, which relates especially to issues 1) and 2).

Phase I: Experimental phase of the PHR. The first identified phase starts in 2004 (01 Jan. 2004) when statutory health insurance companies were legally allowed to finance PHRs to improve quality and efficiency and to gain initial concrete experience in day-to-day healthcare practice provided by physicians. For twelve years (from 2004 to 2016), this self-administration was not in any way centrally managed or steered to provide a clear vision for the PHRs to interface with that of other service providers and to value-adding services to increase the attractiveness of the Telematic Infrastructure (TI). In December of 2018, gematik published the first version of an EHR, which will require an integrated application of the TI (19 Dec.2018). Contrary to expert opinions and European solutions with established standards, e.g., Integrated Healthcare Enterprises (IHE), gematik decided to follow a proprietary noninternationally-standardized approach, which is not interoperable with other existing solutions on a technical or a syntactical level outside of the TI, i.e. with that of other nations. The development of TI was plagued by various problems, delays, inadequate regulations, outdated technologies, etc. (e.g., [30]). These were partly caused by disagreements among the shareholders and stakeholders. Overall, this episode is characterized by the failure of the system of selfadministration. The system could not gain sufficient momentum without hierarchical regulatory guidelines, due to direct and indirect network effects, and thus failed to support widespread EHR/PHR solutions. Vastly different particular interests (e.g., [28]) prevent agreement about necessary standards and specifications. After fourteen years, the government lost patience and intervened to demand greater consistency. In order to accelerate the process of digitalization of the healthcare system, the Federal Ministry of Health (FMH) took over the majority of gematik through the Appointment Service and Care Law (ASCL) (11 May 2019).

During this first phase, initial solutions from German providers as well as internationally dominated PHR platforms (e.g., Google, Apple, etc.), which are also considered influential and relevant by PHR platform ecosystems, became established, despite the risk of as yet unknown standards and regulations.

Phase: II Transition phase from PHR to EHR. With the ASCL (11 May 2019), wide-ranging regulations for EHR were established, including a date for the introduction of technical and infrastructural regulations. Additionally, the responsibilities of interoperability were clearly regulated and assigned according to layers of interoperability. Gematik is in charge of the technical and syntactical specifications of the EHR, especially the infrastructure, and the National Association of Statutory Health Insurance Physicians (ASHIPs) is responsible for establishing the syntactical and semantical specifications for the EHR content. For this purpose, so-called Medical Information Objects (MIO) are defined in order to determine the structure for health documents (i.e., doctor's letter or vaccination certificate etc.) in the EHR. Within this specification, the authorities define the regulatory framework according to issue 2), of Fürstenau et al. [11], regarding the design of the architecture and standards of the EHR. Based on this, the statutory health insurance companies can develop their own EHR/PHR solution, which puts them in direct competition with each other. With the resolution of the Digital Health Service Law (DHSL), Germany is the first country to enable the medical prescription of approved Digital Health Services (DHS) i.e., health apps (07 Nov. 2019) that will be financed by statutory health insurance companies, as is clearly regulated by the digital Health Applications Law (DHAL) (15 Jan. 2020). This allows potential complements (DHS) for the PHRs/EHRs to be supported. The DHSs are also subject to interoperability requirements in order to transfer data to the EHR, which has been enforced by the Patient Data Protection Law (PDPL) (11 Apr. 2020). This law represents a major breakthrough in 
terms of the semantical interoperability to build an interoperable digital healthcare system based on international standards with the acquisition of the license of the international semantic standard SNOMED CT. However, this only refers to the semantic part of coding and the uniform understanding of health information, but does not render the EHR interoperable internationally.

Phase: III EHR becomes mandatory. According to the ASCL, when the EHR will be introduced (01 Jan. 2021), each statutory health insurance company must offer its insurants an EHR that will take effect on January 1, 2021. One year later, the official financial support for PHRs will end. There will only be a single EHR for each insured person, as provided by his/her health insurance company. Additionally, it must be possible for insurants to transfer their data to another health insurance provider in the EHR, if necessary (01 Jan. 2022).

3.4.3. The case of TK and AOK from the meso and micro level point of view. In order to answer the research question, how PHR and their respective ecosystem develop differently over time in a highlyregulated market, we focus in this and the subsequent section on the micro and meso level, i.e., the relevant events during the emergence of the PHR platforms. Initially, the parallels and differences of the incumbent players AOK and TK are described in more detail, whereas the entrant's player VIVY will be described in the next section (3.4.4. ).

Phase I: Experimental phase of the PHR. More than twelve years after the PHR was financially supported, the AOK is establishing a PHR solution (01 June 2016). For this purpose, the AOK, as the largest association of health insurance companies with a total of 26 million insurants, is choosing a decentralized approach, i.e., the data will be stored by the healthcare provider who creates the health data for its patient. In terms of data access and exchange, the AOK is following international standards, particularly IHE integration profiles on technical and syntactical levels. The AOK seeks to establish a health network for the service providers, which relates to issue 3 ) by Fürstenau et al. [11] (13 Sept. 2016 and 10 Oct. 2017).

The second largest health insurance company in Germany with around ten million insurants is also developing a PHR (21 Feb. 2017). In contrast to the $\mathrm{AOK}$, the TK are following a centralized approach to data storage and developing the PHR together with their cooperating company, IBM (IBM Watson, see also [2]), which has many years of experience in datahosting and artificial intelligence (AI) in healthcare. With regard to the above-cited vision of the TK, its cooperation with IBM illustrates the added value that the TK can offer. This enables TK to operate a preventative care management by offering e.g. AIbased value-added services to its insurants. Despite the different strategies and architectures (centralized/ decentralized) relating to issues 1 ) and 2 by Fürstenau et al. [11], the two largest insurance companies, AOK and TK decide to cooperate as co-opetition [27] (11Dec-2018) to enable data exchange among hospitals, insurance companies and PHRs via an interface based on the international IHE standard (11-Apr-2019).

Phase: II Transition phase from PHR to EHR. Following the publication of the specifications by gematik and ASHIP, both insurance companies (AOK $\&$ TK) will be developing an EHR. Given the differences between the existing PHR solutions and the specified EHR in terms of architecture - according to a decentralized approach and interoperability following non-internationally standardized approaches - both platform owners decided to continue to offer their PHRs as an encapsulated solution. With their PHR platform, platform owners can offer complementary services to differentiate their business from the competition, while retaining the flexibility and control according to their own risk aversion (development risks).

Relating to issue 4) Engaging with the platform's ecosystem and wider environment [11], the AOK organized a community event with health startups to expand its PHR ecosystem, and to attract innovative services to their platform, as part of their offering to their insurants (07 Nov. 2019).

Phase: III EHR becomes mandatory. As of January 1, 2021, all statutory health insurance companies are required by law to offer an EHR. One year later, insurants have to be able to transfer their EHR data to another statutory health insurance company, to allow insurants to switch. However, this only refers to interoperability of the specified EHR and not the PHR itself. Thus, some personal data and PHR specific services may not be transferable unless explicitly stipulated by law.

3.4.4. The case of VIVY from the meso and micro level point of view. VIVY and its main shareholder Allianz (70\%), offers a PHR solution for other health insurance companies. With the Allianz-Group, VIVY has an economically powerful partner which is one of the world's leading insurance groups. However, Allianz in Germany focuses on private insurance and asset management products. In contrast to AOK's and TK's solutions, VIVY follows a centralized mobile approach with a greater focus on user experience and the autonomy of the data that comes through storing the data encrypted on the insurants' mobile devices (see also [2]). 
Phase I: Experimental phase of the PHR. The IT service provider of the statutory health insurance companies (Bitmarck) assigned VIVY the contract to provide a PHR for their customers (statutory health insurance companies); therefore, VIVY became a supplier of PHRs for private and statutory insurance companies (01 May 2018). On September 17, 2018, VIVY released its PHR. Consequently, VIVY became the first platform owner of a PHR to provide a nationwide solution for all insurants of the contractual health insurance companies. This triggered network effects and VIVY quickly acquired additional health insurance companies (22 July 2019). At the time of writing (Oct. 2020), VIVY's ecosystem includes twenty-nine statutory health insurance companies and four private health insurance companies, each with their own instance of PHR. A potential 19,4 million insurants can use their PHR. Additionally, VIVY is cooperating with several hospitals on the expansion of their ecosystems (12-Aug-2019).

Phase: II Transition phase from PHR to EHR. After the announced specification and date of the EHR, VIVY will not offer an official EHR for statutory health insurance companies. Its strategy for this decision remains unclear, but may be based on the large architectural differences between its product and the general EHR specifications and regulatory requirements, or based on the fact that the customer group of the statutory insured do not fit the strategic focus of its shareholders. Therefore, the contract has been awarded to the provider RISE. Nevertheless, VIVY will still be seen by the contract partner as a provider of innovative solutions to connect complementors e.g., digital health applications such as Digital Health Service (DHS), and to offer innovative health services via their mobile PHR solution.

\section{Discussion}

In order to answer the research questions, the key events in the emergence of the EHRs and PHR platforms were structured into phases and levels to show - on the basis of longitudinal data- how the ecosystems of the PHR platforms emerge. As a result, interoperability challenges at single points of time could be derived from the perspective of the respective stakeholders.

Platform owners' point of view: An initial challenge is the appropriate 1) timing of designing technical architecture and selecting standards, reflecting issue 2) by Fürstenau et al. [11]. During Phase I, the three platform owners have demonstrated a possible PHR design, showing how an EHR could potentially be structured in order to enter the

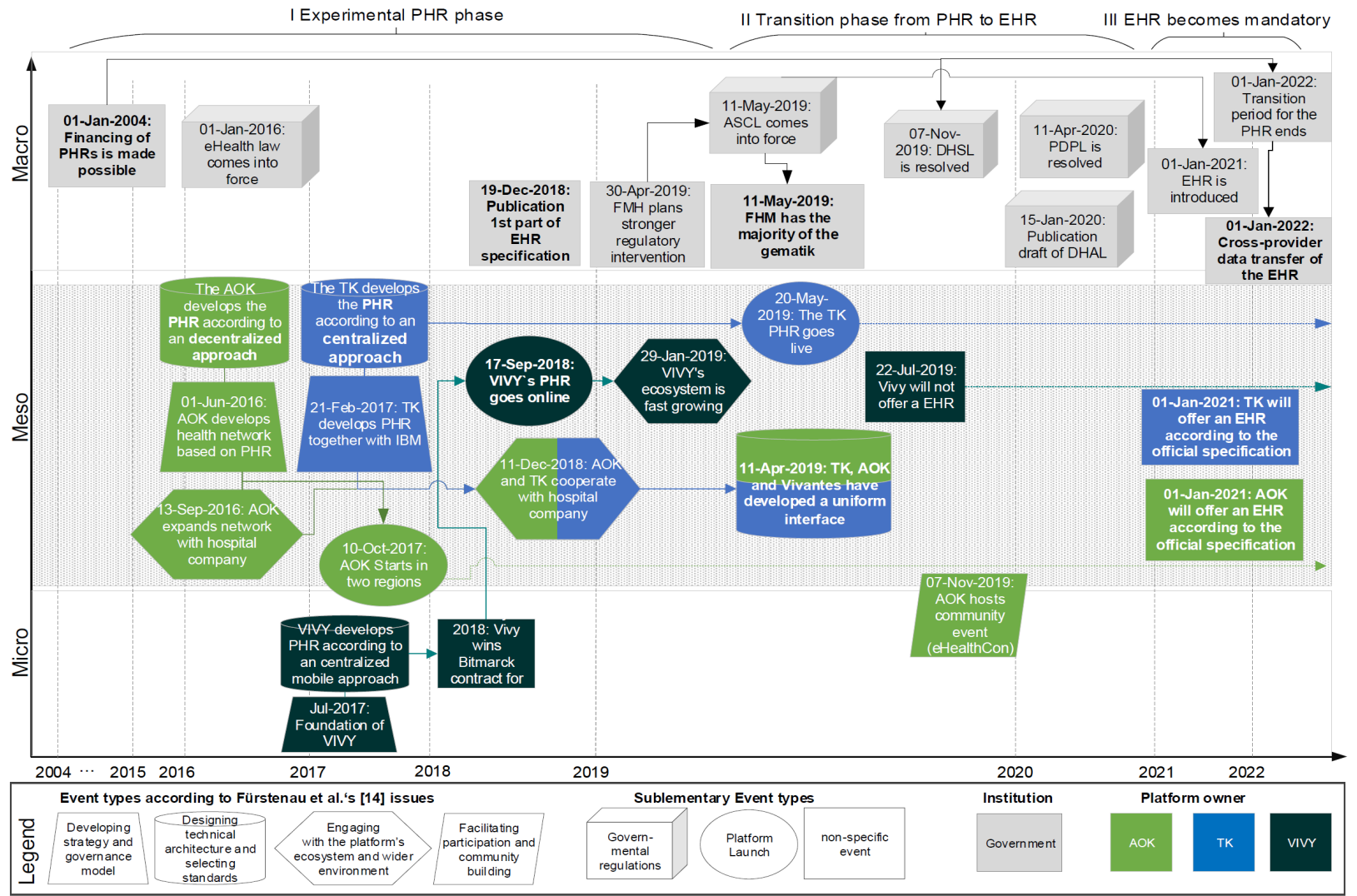

Figure 1. Emergence and dynamics of three PHR platforms in Germany 
discussion about technical and syntactical interface specifications on the macro level with gematik and the Association of Statutory Health Insurance Physicians, among others, during the transition to Phase II. This gives providers the opportunity to start diffusion processes at an early stage to promote network effects around their respective solutions and emerging ecosystems. At the same time, there is an investment and 2) the development risk of interfaces, especially in times of uncertainty regarding the final specifications. The uncertainty that characterized the transition from phase I to phase II is likely to have resulted in costs due to adjustments that had to be made to the interfaces, especially on a technical and a syntactical level. The platform owners AOK and TK have shown how an interface can work according to international standards, as it was to be expected that gematik would follow these standards. However, the published specification of the EHR does not follow IHE, the established international standard. Due to the high level of adaptation of the existing system, AOK and TK decided to develop separate solutions according to the national EHR specification. Therefore, the PHR from AOK and TK will be offered as an additional MSP-offering that fulfill the regulative basic affordances of an EHR but maintain the control and flexibility of the platform while promoting innovation and avoiding further development risks for their ecosystem. In contrast to the EHR, the platform owners of the PHR are free to make decisions about the design of the interfaces. This relates to issues 1) and 3) by Fürstenau et al. [11], and the degree of openness [31] via interfaces [27]. Platform owners have to 3) choose the appropriate Governance Mode [26] on the meso and micro levels of the platform's ecosystem for co-opetition with competitors, and cooperation with complementors [27] regarding interoperability requirements to promote innovations, e.g., by complementors such as Digital Health Service (DHS). The case of the co-opetition with the two incumbents AOK and TK shows that two competitors with partly different customer groups and a different platform architecture can cooperate in order to avoid being displaced by new entrants and to build solid and partly interoperable PHR platforms.

Complementors' point of view: This case also highlights the challenges for complementors. This includes 4) interoperability as a financing criterion of digital health services e.g., health startups, to be approved as (DHS) in the context of Germany. Therefore, interoperability has to be an integral part of the strategy to get financial support from statutory health insurance companies. Furthermore, interoperability via standard interfaces is a basic requirement of complementors connecting to
PHR/EHR platform ecosystems [3, 4, 10, 11]. In Germany, this concerns at least five health startups during Phase II that have officially approved as DHS (fifteen have applied and will be reviewed, status as of November 2020) in order to be prescribed by physicians and reimbursed by the insurance companies. The different architectures of the platforms and governance modes of the platform owners result in another challenge for the complementors, namely to also 5) choose the appropriate platform in terms of interoperability. In addition to criteria relating to the size of the ecosystem and to potential users of the platforms, interoperability considerations are crucial, e.g., with regard to the type and nature of the data/information needed from the EHR and/or PHR. The adaptation effort to the PHR platform would be relevant in this context for the complementors. From a technical/syntactical point of view, the complementors has to determine whether the service requires the data/information from the EHR, e.g., doctor's reports, examination results, or from the PHR, such as self-collected vital parameters by the insurants. AI-based services, for instance, as probably intended e.g. by TK with its partner IBM Watson require more stringent interoperability requirements and semantic standards than less extensive services [21]. Based on well-coded health data/information, e.g., SNOMED CT, AI-based services can deliver better results [21]. Another factor arises among the different architectures of the platform (centralized/ decentralized) and respective access via e.g., standardized or less standardized interfaces - the amount of effort necessary for the integration depends on this. The respective adjustments and the threat of switching costs (for additional platforms, etc.) results in 6) binding effects for the complementors. Platform owners try to avoid multihoming, for users as well as complementors [29]. Detailed solutions within and between the emerging platform ecosystems, especially on a technical level, will be too different, e.g., in the case of VIVY. The health data would be stored directly on the device and could be accessed e.g., by Appbased services provided by the complementors, whereas in the case of AOK, the data would be stored in various decentralized IT infrastructures and would have to be retrieved first. This included necessary specifications for the concrete design and linking of business and supply processes, the coding of treatment and billing details, the supplementation of the regulatory EHR mandatory elements, and additional possibly ecosystem-specific value-added services.

Insurants' point of view: The case also shows some challenges for platform users, including the patients/insurants. When an insurant decides to switch their health insurance company, she/he can transfer 
her/his EHR-specified data to the platform of the new statutory insurance company (from January 1, 2022). However, this does not apply to the additional data held in the PHR. Depending on the insurants' personal data and additional, in some cases PHRexclusive/specific services, switching costs will result. This can lead to 7) lock-in effects to the initial platform and thus to the respective statutory health insurance company, effectively preventing multihoming by the platform owners [34]. This aspect would also be interesting regarding whether and what kind of role the emerging tech platforms and ecosystems of Apple, Google or Amazon, etc., will play and whether and how their health services can be tackled, integrated or combined with the respective PHR strategies by the incumbents, as mentioned by Thomas Ballast in his statement (p. 1). Apple, for example, is using the FHIR standard to integrate further health services, and it remains to be seen whether this will also apply to other record solutions.

\section{Limitations and outlook}

The analysis and discussion of the case-study has certain limitations and carries implications for further research. In this study, the management and design framework by Fürstenau et al. [11] was applied from an external point of view, i.e., in some cases detailed background information would be necessary to elaborate on further instances. For the structuring of the events, three supplementary event types (governmental regulations, platform launch and nonspecific event) were used (see Figure 1). Some of the identified aspects are closely linked to this specific German case and are therefore not generally valid, which would have to be examined individually in future studies.

As a brief outlook for this case, three aspects merit being further examined in future research. First, in response to the initial TK quote, it will be interesting to see if and how the relationship between the incumbent ecosystems and international tech giants like Google, Apple and Amazon will develop, considering that they have stated their intention to target health as their next big frontier. A "battle of the platforms" can be expected, which will be shaped by network effects and the decisions of complementors and insurants as well as regulators in response to the chosen strategies of the respective platform owners. Second, platform owners have to compete with each other for domination of the PHR/EHR market segment from 2021 onwards. The effects their chosen strategies will have on the attraction of complementors would represent another avenue for future study, particularly concerning platform architectures. Third, it would help to understand the internal view of complementors, including what challenges they perceive and how they deal with them. The existing demand for technical and economic strategies and principles during the emergence of EHR and PHR platforms in order to handle these challenges should be addressed in further research, e.g., following a design-oriented approach.

\section{Conclusion}

The study makes the following contributions: First, in contrast to what we know about interoperability and platforms as pre-conditions $[3,4,10]$ and design parameters $[11,19]$, especially in terms of openness [27], this study reveals the central role played by interoperability as a design parameter for emerging MSPs, and contributes to the interoperability discussions of MSPs, especially in healthcare. As part of this we identified seven key challenges for stakeholders, which are: For platform owners: 1) the timing of designing technical architecture and selecting standards, especially in periods of high uncertainty, especially 2) to avoid the development risk of interfaces and resulting adjustments; 3) platform owners have to choose the appropriate governance mode to balance interface openness, e.g., with competitors and complementors. 4) Interoperability can be a criterion for funding or reimbursement and should form part of their strategy, especially for complementors. 5) Complementors also have to choose the appropriate platform to generate interoperability i.e., interfaces and data composition. 6) Proprietary adaptations to an ecosystem can also lead to binding effects. 7) From the perspective of the insurants, there are also challenges resulting from the lack of interoperability between PHR and other platforms, which may result in lock-in effects for the insurants to various, perhaps converging platforms of incumbents and entrants in health as one of the next big "digital transformation battle fields". Second, a discussion and comparison between PHR platforms and the EHR points out the strategic differences between the three providers, which also leads to divergent architectural and interoperability challenges for complementors, insurants, and not least for platform owners themselves. Third, the study provides an overview of the significant changes in the German healthcare system triggered by digitalization and the emergence of MSP platforms and the EHR. Fourth, the discussion about the challenges has implications that can inform researchers as well as insurance companies or technical health service provider e.g. of health platforms, in Germany and also in other countries. 


\section{References}

[1] Aanestad, M., M. Grisot, O. Hanseth, and P. Vassilakopoulou, "Strategies for Building eHealth Infrastructures", in Information Infrastructures within European Health Care, M. Aanestad, M. Grisot, O. Hanseth, and P. Vassilakopoulou, Editors. 2017. Springer International Publishing: Cham $(\mathrm{CH})$.

[2] Auschra, C., M. Gersch, T. Kurth, and C. Vogt, Path creation as a strategy to manage uncertainties of digitalization: German health insurances and the set-up of digital health platforms, Tallinn (Estonia), July 5- 72018.

[3] Benson, T. and G. Grieve, eds., Principles of Health Interoperability, Springer Publishing, Cham $(\mathrm{CH}), 2016$.

[4] Blechman, E.A., P. Raich, W. Raghupathi, and S. Blass, "Strategic Value of an Unbound, Interoperable PHR Platform for Rights-Managed Care Coordination", Communications of the Association for Information Systems, 30, 2012.

[5] Boudreau, K. and A. Hagiu, "Platform Rules: MultiSided Platforms as Regulators", in Platforms, markets and innovation, A. Gawer, Editor. 2009. Edward Elgar: Cheltenham.

[6] Bowden, T. and E. Coiera, "The role and benefits of accessing primary care patient records during unscheduled care: a systematic review", BMC Medical Informatics \& Decision Making, 17(138), 2017.

[7] Busse, R., M. Blümel, F. Knieps, and T. Bärnighausen, "Statutory health insurance in Germany: a health system shaped by 135 years of solidarity, self-governance, and competition", The Lancet, 390(10097), 2017, pp. 882-897.

[8] Cusumano, M., "Technology strategy and management: The evolution of platform thinking", Communications of the ACM, 53(1), 2010, pp. 32-34.

[9] Eisenhardt, K.M., "Building Theories from Case Study Research", The Academy of Management Review, 14(4), 1989 , p. 532.

[10] Essén, A., I. Scandurra, R. Gerrits, G. Humphrey, M.A. Johansen, P. Kierkegaard, J. Koskinen, S.-T. Liaw, S. Odeh, P. Ross, and J.S. Ancker, "Patient access to electronic health records: Differences across ten countries", Health Policy and Technology, 7(1), 2018, pp. 44-56.

[11] Fürstenau, D., C. Auschra, S. Klein, and M. Gersch, "A process perspective on platform design and management: evidence from a digital platform in health care", Electronic Markets, 29, 2019, pp. 581-596.

[12] Gawer, A. and M.A. Cusumano, "Industry Platforms and Ecosystem Innovation", Journal of Product Innovation Management, 31(3), 2014, pp. 417-433.

[13] Ghazawneh, A. and O. Henfridsson, "Balancing platform control and external contribution in third-party development: the boundary resources model", Information Systems Journal, 23(2), 2013, pp. 173-192.

[14] Hanseth, O., E. Jacucci, M. Grisot, and M. Aanestad, "Reflexive Standardization: Side Effects and Complexity in Standard Making", MIS Quarterly, 30, 2006, pp. 563-581. [15] Hein, A., M. Schreieck, T. Riasanow, D.S. Setzke, M. Wiesche, M. Böhm, and H. Krcmar, "Digital platform ecosystems", Electronic Markets, 43(1), 2019.

[16] Herrmann, M., P. Boehme, T. Mondritzki, J.P. Ehlers, S. Kavadias, and H. Truebel, "Digital Transformation and
Disruption of the Health Care Sector: Internet-Based Observational Study", Journal of medical Internet research, 20(3), 2018, e104.

[17] IEEE StandardS Board, "Interoperability", in IEEE standard glossary of software engineering terminology, IEEE StandardS Board, Editor. 1990. Institute of Electrical and Electronics Engineers: New York.

[18] Kapoor, R., "Ecosystems: broadening the locus of value creation", Journal of Organization Design, 7(1), 2018.

[19] Kohli, R. and S.S.-L. Tan, "Electronic Health Records: How Can IS Researchers Contribute to Transforming Healthcare?", MIS Quarterly, 40(3), 2016, pp. 553-573.

[20] Langley, A., "Strategies for Theorizing from Process Data", The Academy of Management Review, 24(4), 1999, pp. 691-710.

[21] Lehne, M., J. Sass, A. Essenwanger, J. Schepers, and S. Thun, "Why digital medicine depends on interoperability", NPJ digital medicine, 2(79), 2019.

[22] Markus, M.L. and D. Robey, "Information Technology and Organizational Change: Causal Structure in Theory and Research", Management Science, 34(5), 1988, pp. 583-598. [23] Obermann, K., P. Müller, H.-H. Müller, and B. Glazinski, eds., The German health care system: Accessing the German health care market, 2nd edition, medhochzwei, Heidelberg, 2016.

[24] OECD, Health at a Glance 2019, Retrieved from: https://www.oecd-ilibrary.org/docserver/4dd50c09-en.pdf, Accessed: June 1st 2020.

[25] Oemig, F. and R. Snelick, eds., Healthcare Interoperability Standards Compliance Handbook, Springer International Publishing, Cham (CH), 2016.

[26] O'Mahony, S. and R. Karp, "From proprietary to collective governance: How do platform participation strategies evolve?", Strategic Management Journal, 2020.

[27] Ondrus, J., A. Gannamaneni, and K. Lyytinen, "The Impact of Openness on the Market Potential of Multi-Sided Platforms: A Case Study of Mobile Payment Platforms", Journal of Information Technology, 30(3), 2015, pp. 260275.

[28] Stegemann, L. and M. Gersch, "Interoperability Technical or economic challenge?", it - Information Technology, Vol. 61(5-6), 2019, pp. 243-252.

[29] Tiwana, A., Platform ecosystems: Aligning architecture, governance, and strategy, Morgan Kaufmann, Amsterdam, 2014.

[30] Wessel, L., M. Gersch, and E. Harloff, "Talking Past Each Other", Business \& Information Systems Engineering, 59(1), 2017, pp. 23-40.

[31] Witte, A., D. Fürstenau, and R. Zarnekow, "Digital Health Ecosystems for Sensor Technology Integration - A Qualitative Study on the Paradox of Data Openness", 41st International Conference on Information Systems (ICIS) 2020 At: Hyderabad, India, forthcoming.

[32] Yaraghi, N., A.Y. Du, R. Sharman, R.D. Gopal, and R. Ramesh, "Health Information Exchange as a Multisided Platform: Adoption, Usage, and Practice Involvement in Service Co-Production", Information Systems Research, 26(1), 2015, pp. 1-18.

[33] Yin, R.K., Case study research and applications: Design and methods, 6th ed., SAGE, Los Angeles, 2018. 